\title{
Multicast Address Allocation Based on Interest Constraints
}

\author{
Yunxia $\mathrm{Yu}$ \\ College of Computer Engineering \\ Jingchu University of Technology \\ Jingmen, P.R.China \\ 11440812@qq.com
}

\author{
Weiqun Han \\ School of Automobile Engineering \\ Wuhan University of Technology \\ Wuhan, P.R.China \\ Hanweiqun01@sohu.com
}

\author{
Xiaoxuan Tian \\ Control and Electronic Technology \\ Beijing Research Institute \\ Beijing, P.R.China \\ tianjingxuan@sina.com
}

\begin{abstract}
Multicast address allocation is a key and difficult points of HLA simulation system. Multicast address allocation need to select or improve according to the actual application needs. In the data distribution management based HLA simulation grid, for the contradiction of redundant data receives and multicast address demand, we put forward a multicast address allocation of HLA hierarchy structure and described the data forwarding algorithm. Within the site, multicast address allocation use the method based on the data receiver and forwarding server contains a filter allocation table based on the receiver's multicast address. When there is a data access, RTIA according to assign tables to forward the data to the appropriate multicast addresses. Using this method, you can avoid node receives data not related to.The simulation experiment and results analysis show that: the maximum level of nodes in the multicast reception of the message overhead can be significantly enhanced; using the hierarchical multicast routing aggregation, and based on the aggregation of routing information that is both feasible and effective.
\end{abstract}

Index Terms -Distributed Simulation, Multicast, Address Allocation, Hierarchy Structure .

\section{INTRODUCTION}

In a large-scale distributed simulation systems, the main means of communication is used for multicast. Multicast address allocation is a key and difficult points of HLA simulation system, it is also the key technology in establishing channel of interested management. It is a pair of sharp contradictions that the huge demand for multicast addresses in distributed simulation system and the limitation of routers, a host can support the multicast address.

In General, we can use the following map to match the relationship between a data source and destination after the completion calculation.

$$
M\left[S, \bigcup_{i=1}^{n} d_{i}\right]
$$

In the above maps, the $S$ means a data source, the $d_{i}$ means destination of i-node. The work mainly of multicast communication in the following three areas: assign a multicast address for the above maps; sent on the data to the multicast address; boot that correspond to the nodes on the machine to join to the allocated multicast address.

In a distributed simulation systems, multicast address allocation method can be divided into the following three categories:

(1)The multicast address allocation method based on data content. Grid distribution method is to use this multicast address allocation methods. In the DIVE system, the scene hierarchy tree is divided into more than one light weight group, and to assign a multicast address. In the NPSNET-IV system, the terrain mesh is divided into some grid, and to assign a multicast address. The multicast address allocation method has the advantage of small amount of calculation and Its disadvantage is that when the simulation node increases, corresponding multicast address demand will grow rapidly, causing a multicast address large amounts of waste. To avoid rapid increases in demand and waste of multicast addresses, A.Boukerche make a centralized dynamic multicast address allocation policy. But its shortcomings are due to the centralized distribution, its central server is likely to be a bottleneck in the simulation process.

(2) Multicast address allocation method based on the data received. Such allocations each of the target node set is assigned a multicast address. Depending on the target node list, data sent to the multicast address of the target node. Advantage of this method is: each simulation nodes do not receive the data that it don't want. Simulation system for interconnection with $\mathrm{N}$ nodes requires $2^{N}-1$ multicast address. To reduce the number of multicast addresses, each simulation node can only define a multicast address that contains all nodes, and target node using a fixed number of multicast addresses are maintained, but the problem is: filter efficiency decreased.

(3) Multicast address allocation method based on optimal problem solving. Scholars such as M.Adlery believe that communication can be broken down into two issues: relation between data source and multicast addresses, recipients and multicast address of correspondence. He gives a random assignment method for access to secondbest, and evidence that the recipient receives the minimize the amount of unnecessary data and general communication is an NP complete problem. Advantages of this method of address assignment is: through the optimal treatment, makes very little wasted multicast address. Problem is: dynamic maintenance of global communication, communication costs more.

In summary, three kinds of multicast address allocation method has its advantages and disadvantages of the first two is the need for a large number of multicast addresses, 
disadvantage of the third is that traffic is too large. Multicast address allocation in simulation grid system, need to select or improve according to the actual application needs.

\section{II . Multicast AdDRess Allocation Of Hierarchy}

(1)Multicast address allocation structure model based hierarchy

In the data distribution management based HLA simulation grid, for the contradiction of redundant data receives and multicast address demand, we put forward a multicast address allocation method of hierarchy. Its structure model as shown in Fig.1.

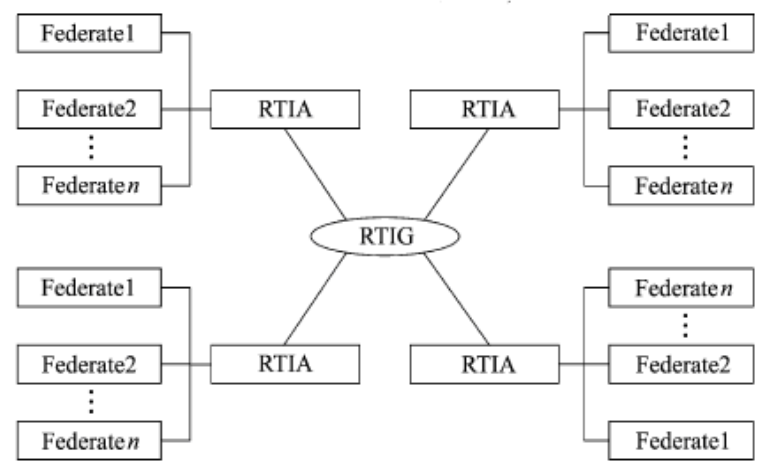

Fig.1. Multicast address allocation model based hierarchy.

In the structure of the model, RTIA(RTIAmbassador) on the local server attached to the various members of the Federal and the communication mode is UNIX Socket. By TCP (UDP), RTIG(RTIGateway) interacts with the RTI and the RTIA is not established connections directly.

The functionality of RTIA includes the following two main areas:

(1)Receive the local federal Member data from RTIG and send the local federal Member data to RTIG.

(2) Match calculation of regional and multicast address allocation.

(2) Basic principles of multicast address allocation based hierarchy .

In the multicast address allocation based hierarchy, the data filtering methods are as follows: The federal member of RTIA, its multicast address allocation method based on the recipient's; For each RTIA, the multicast address allocation method of filter based on the sender.

For each local RTIA, It is capable of recording the descriptive information and filter information. Inside the RTIA $_{i}$,if exists intersection between the federal order area and release area, RTIA $_{i}$ assigned multicast addresses based on recipient filtering and received the internal data of the members of the federal. For RTIA $\mathrm{A}_{j}$ if federate members need the data, then RTIA $_{j}$ join to the RTIG multicast address and receives data. I .

In RTIA $_{\mathrm{j}}$, forwarding of data format as shown in table
TABLE I

FORWARDING FORMATS OF DATA

\begin{tabular}{cc}
\hline data source & send channel \\
\hline$\langle$ site, federate, entity $\rangle$ & $\langle$ multicast address $\rangle$ \\
\hline
\end{tabular}

In the above table, when a multicast address is not null, indicates an external receiver, then the data posted by RTIG to multicast address corresponding to the site.

For RTIA $\mathrm{f}_{\mathrm{j}}$ that accepts data, the data format as shown in table II.

TABLE II

RECEIVING DATA FORMAT

\begin{tabular}{cll}
\hline data source & the receiving node & send channel \\
\hline $\begin{array}{c}\text { site , federate, entity } \\
>\end{array}$ & $\sum_{i=1}^{n}$ Federate $i$ & $\begin{array}{l}\langle\text { multicast } \\
\text { address }\rangle\end{array}$ \\
\hline
\end{tabular}

For RTIG, as soon as it receives data from RTIA $_{i}$, the collection and multicast addresses can be get by table. Subsequently, the multicast addresses of RTIA $\mathrm{j}_{\mathrm{j}}$ are sent to the receiving node in the site.

The above multicast address allocation architecture, has the following advantages: Using the multicast address assignments based on data sent between sites, filtering and forwarding of data by RTIG based on filter match results, can ensure that data of independent nodes is not received; Within the site based on the data received multicast address allocation scheme, as a grouping node, you can avoid the combinatorial explosion problem.

\section{Multicast AdDRESS Allocation AndDatA FORWARDING ALGORITHM}

Through the above analysis, we can see: regional matches are is the base of multicast address allocation and forwarding data.

For servers on a site i, on which the filter can be divided into two categories.

(1)The information of internal filter

The information of internal filter is mainly used to record site internal filter information of the members of the federation. Its published information and ordering information can be expressed as follows, respectively: $<F_{i}^{l}, P_{i}^{l}>;<F_{i}{ }^{l}, S_{i}^{l}>$ 。

(2)The information of external filter

The information of external filter is mainly used to record external site filter information. Its published information and ordering information can be expressed as follows, respectively: $<$ RTIA $_{\mathrm{j}}, \sum_{m=1}^{n} P_{j}^{m}>;<R T I A_{j}, \sum_{m=1}^{n} S_{j}^{m}>$.

Below, we give a multicast address allocation and data forwarding algorithm on filter forwarding server.

/*Initialization of a multicast address allocation*/

For each RTIAj, a multicast address is assigned

$/ *$ Generates three kinds of collections. Set ${ }_{i}^{l}$ indicates that the receiving node collections; Out ${ }_{i}$ indicates an external receiving station collection; $R c v_{i}^{j}$ indicates that the internal node collection of receive external sites $\mathrm{j}$.

$$
\operatorname{Set}_{i}^{l}, \text { Out }_{i}, \operatorname{Rcv}_{i}^{j}=\varnothing ; / * \text { initialization*/ }
$$




$$
\begin{aligned}
& \operatorname{Set}_{i}^{l}=S_{i}^{l} \cap \sum_{m=1}^{n_{i}} P_{i}^{m} \quad\left(m \neq l, l=1, \ldots, n_{i}\right) ; \\
& \text { Out }_{i}=\sum_{m=1}^{n_{i}} P_{i}^{m} \sum_{m=1}^{n_{j}} S_{j}^{m}(j=1, \ldots \ldots, N) ; \\
& \operatorname{RCV}_{i}^{j}=\sum_{m=1}^{n_{i}} S_{i}^{m} \sum_{m=1}^{n_{j}} P_{j}^{m}(j=1, \ldots \ldots, N) \circ \\
& / * \text { multicast address allocation*/ }
\end{aligned}
$$

(1) If $\operatorname{Set}_{i}^{l} \neq \varnothing, \operatorname{RTIA}_{i}$ allocate multicast addresses based on the data receiver by Set $_{i}^{l}$ generating method.

(2)If $R c v_{i}^{j} \neq \varnothing, R T I A_{i}$ allocate multicast addresses based on the data receiver by $R c v_{i}^{j}$ generating method.

/*To send data to an external site*/

(1) If $O u t_{i} \neq \varnothing$ and $\operatorname{Set}_{i}^{l}=\varnothing$, boot $F_{i}^{l}$ to send data to the address $M C_{i}$ of corresponding to RTIA $_{i}$.

(2) If Out $_{i} \neq \varnothing$ and $\operatorname{Set}_{i}^{l} \neq \varnothing$, then $R T I A_{i}$ join to address $M C_{x i}$,and forward the data to the address $M C_{i}$

/* Data that is received from an external site*/

If $R c v_{i}^{j} \neq \varnothing, R T I A_{i}$ will join to the multicast address of corresponding to the external site $R c v_{i}^{j}$, and receives external data.

From the above multicast address allocation algorithms and data forwarding, we can see: Within the site, multicast address allocation use the method based on the data receiver and forwarding server contains a filter allocation table based on the receiver's multicast address. When there is a data access, RTIA according to assign tables to forward the data to the appropriate multicast addresses. Using this method, you can avoid node receives data not related to.

\section{SimUlation EXPERIMENT AND RESUlt ANALYSIS}

Our experiments are divided into two groups. In the first study, the Group's average arrival rate fixed is set to 0.5 , group member changes; In the second study, fixed number of members is set to 15 , the Group's average arrival rate changes. In both sets of experiments, grid load change is achieved through a number of members of the group or the change of groups average arrival rate. We need to draw is the performance parameter changes of the plane structure's and hierarchy structure's.

We define the following parameters:

Grid load $=$ the number of members of the group $*$ average bandwidth $*$ the average arrival rate of the group $*$ the average duration of the group.

Group members take up bandwidth=the bandwidth on reserved multicast tree path/the bandwidth of all requests to join the group members.

Message overhead as reception capacity maximum $=$ the number of the group as reception capacity maximum/ run time.
Bandwidth congestion rate $=$ the bandwidth failed to request to join group members/the bandwidth all requests to join the group members.

In the experiment, under the change conditions of the members number of the group and group arrival rate, we compare their message overhead of plane multicast and hierarchy multicast, the results as shown in Fig.2.

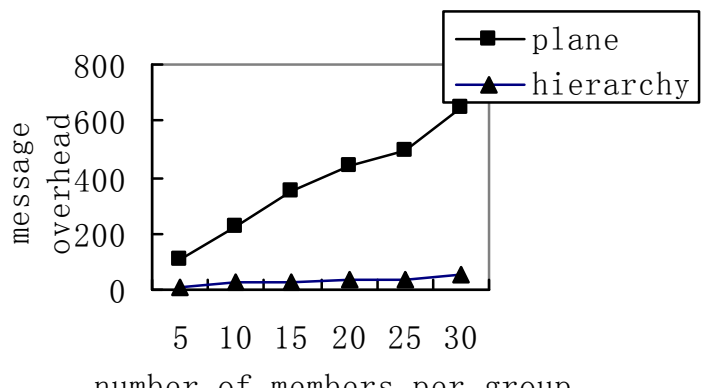

number of members per group

(a) message overhead wi th group member shi $p$ changes

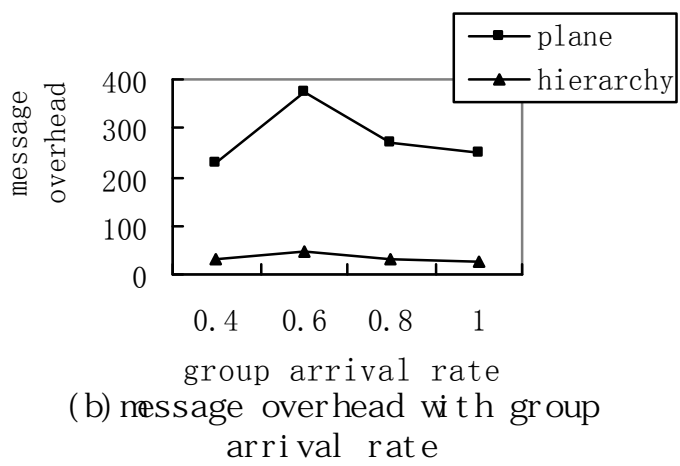

Fig.2. the maximum receive message overhead under different network layer multicast tree node

The experiment shows that: in a distributed simulation, using the hierarchical multicast can muster and limit the range of nodes of information received and sent, can also effectively reduce the amount of information exchanged between routers on multicast tree.

Under normal circumstances, routing algorithm running on a plane multicast based on network-wide information of source, its routing performance is usually very good. Here, we compare routing performance run on the plane multicast and on hierarchical multicast. It aims to test the hierarchical multicast reduces the amount of information exchanged between routers on multicast tree at the same time, will reduce the performance of routing?

In fig. 3 , we give the bandwidth congestion rate changes of plane multicast and hierarchical multicast as the group membership changes. The reasons bandwidth congestion rate increased with the increase in the number of members of the group are: increased number of members of the group, the grid load increases, causing application cannot participate in group members increase, bandwidth congestion rate increases. 


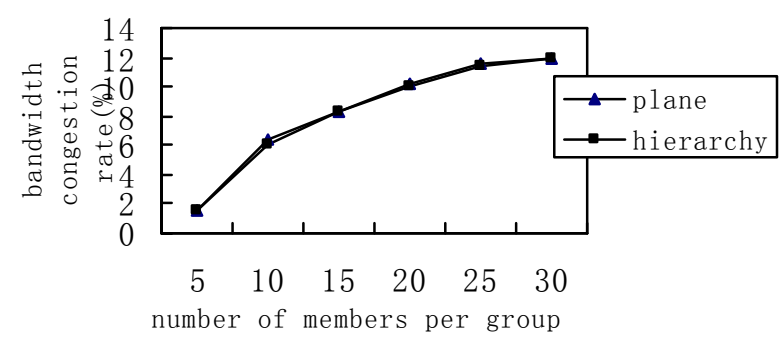

(a) the bandwi dth congesti on $r$ ate changes wi th group member shi $p$ changes

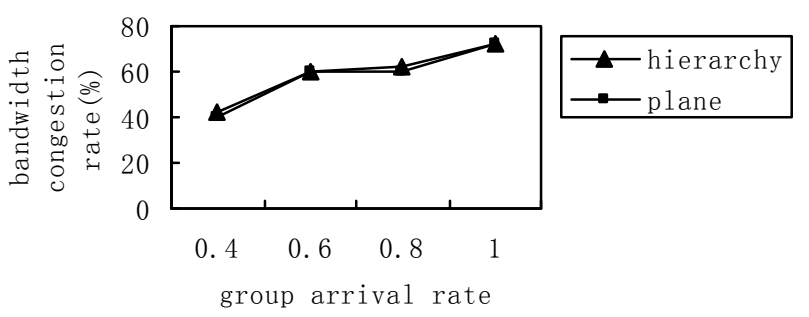

(b) the bandwi dth congest i on $r$ at e changes with group arrival $r$ ate

Fig.3.the bandwidth congestion rate comparison under different network layer

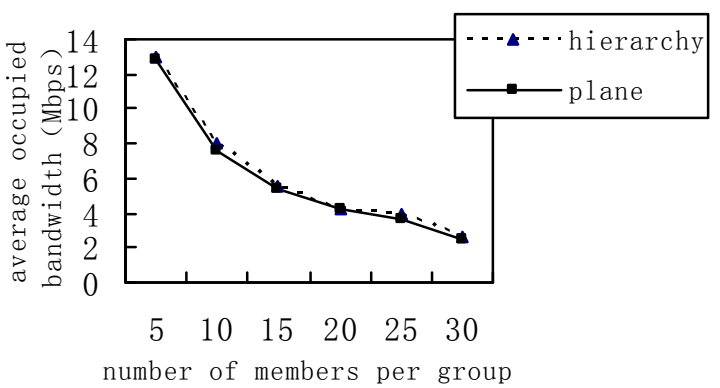

(a) aver age occupi ed bandwi dth with the gr oup member shi $p$ changes

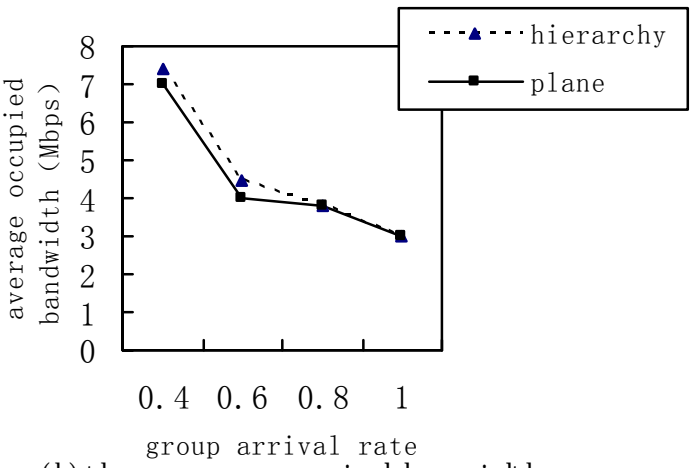

(b) the aver age occupi ed bangwi dth

changes wi th group arrival $r$ ate

Fig.4.the bandwidth occupied changes comparison under different network layer

From fig. 3 (a) ,we can see that bandwidth congestion rate in hierarchical multicast is higher than plane multicast's, but it makes little difference. From fig.3 (b), we can see that when the group arrival rate increases, the grid loads increase, lead to bandwidth congestion rate increases accordingly. From fig.3, we can conclude that: bandwidth congestion rate does not significantly worse because of hierarchy.

In fig.4, we show the average occupied bandwidth changes comparison as the number of group members and the group arrival rate changes. We can see that: the average occupied bandwidth in the hierarchical multicast is higher than in the plane multicast, but the difference is not significant.

From fig. 3 and fig.4, we can see that under variation of load in grid, the bandwidth congestion rate and average occupied bandwidth in hierarchical multicast not much change than in plane multicast. In other words, using the hierarchical multicast routing aggregation, and based on the aggregation of routing information that is both feasible and effective.

\section{ACKNOWLEDGMENT}

This work was supported by the national science foundation of China (item No. 60234030) and the national science foundation of China (item No. 60404021).

\section{REFERENCES}

[1]Wang Bin, Hou J.C.. Multicast routing and its QoS extension:Problems, algorithms, and protocols. IEEE Network, 2000, 14(1):22 36

[2] Lui K.-S., Wang J., Xiao L,et al. QoS multicast routing with heterogeneous receivers. In: Proceedings of the IEEE GLOBECOM, San Francisco, 2003, 3597 3601

[3] Boukerche A, Roy A, Thomas N. Dynamic grid-based multicast group assignment in data distribution management. Proceedings of 4th Workshop on Distribution Simulation and Real-time Application. New York U. S. A.:2000.47 54

[4] Calvin J, Cebula D P, Chiang C J, et al. Data subscription in support of multicast group allocation.In:Proceedings of the 13th Workshop on Standards for the Interoperability of Distributed Simulations.Orlando, FL, 1995. $367 \sim 369$

[5] Katherine Lee Morse. An Adaptive. Distributed Algorithm for Interest Managemene:(Ph D Disseriation). University of California.2000.79 83

[6] K. Czajkowski and A. Dan and J. Rofrano and S. Tuecke and and M. Xu Agreementbased Grid Service Management (OGSI-Agreement). Global Grid Forum, GRAAP-WG Author Contribution Draft, June 2003.

[7] R. Al-Ali, K. Amin, G. von Laszeswski, O. Rana, and D. Walker. An OGSABased Quality of Service Framework. In Proceedings of the Second International Workshop on Grid and Cooperative Computing (GCC2003), Shanghai, China, 2003.

[8] Joshy Joseph,Craig Fellenstein, Grid Computing, IBM Press 2004

[9] X. Tang et al., "QoS Provisioning Using IPv6 Flow Label In the Internet, " Proceedings of the 2003 Joint Conference of the Fourth International Conference on Information, Communications \& Signal Processing and Fourth Pacific-Rim Conference on Multimedia, vol. 2, pp. 1253 - 1257, 15-18 Dec. 2003. 